Classification

Physics Abstracts

$61.16 \mathrm{D}-64.70-68.40$

\title{
In-situ observation and the thermodynamical analysis of the melting process of In particles embedded in an Al matrix
}

\author{
K. Sasaki and H. Saka \\ Faculty of Engineering, Nagoya University, Furo-cho, Chikusa-ku, Nagoya, 464-01, Japan \\ (Received February 8, 1993; accepted March 26, 1993)
}

\section{Introduction.}

Crystalline solid-liquid and solid-solid interfaces play an important role in controlling properties of materials [1]. Between the dimensions on an atomic scale and the dimensions which characterize bulk material is to be found a size range where the surfaces and interfaces control the phase transformation of materials. The melting of microcrystals at temperatures below the bulk melting temperature was first demonstrated by Takagi [2]. The experimental and theoretical works after the Takagi's work have pointed out an important role of the properties of the surface and interface [3-5] in the melting phenomenon of microcrystals. In order to reveal the role of the interfaces during the melting process on In particles embedded in an $\mathrm{Al}$ matrix, an in-situ heating experiment in a high resolution/high voltage electron microscope was performed [6].

\section{Experimental procedures.}

The Al-In system has a monotectic system [7]. The mutual solubility between In and Al is very small below $300^{\circ} \mathrm{C}$. Thus, the processes of the melting and freezing of 'pure' In dispersed in an $\mathrm{Al}$ matrix could be observed. An Al-4.5at\% In alloy was prepared by splat-quenching from nominally pure $(99.999 \%)$ starting materials of $\mathrm{Al}$ and In [8]. TEM specimens were prepared by standard electro-polishing.

The TEM observation was performed in Hitachi H-1250ST operating at $1000 \mathrm{kV}$. The specimen was observed on a double tilting and heating holder. The temperature of the specimen in the electron microscope was kept just below the melting point of In until the drift of the specimen became negligibly small. Then, additional heating was introduced by focusing the electron beam in a small selected area, leading to the melting of the In particles in that region. Lattice fringes of In and $\mathrm{Al}$ were observed in the whole process of melting as shown in figure 1 . The disappearance of moire' fringe between In and Al matrix following the melting of In (Fig. 1) was used to identify the region of the molten In. 

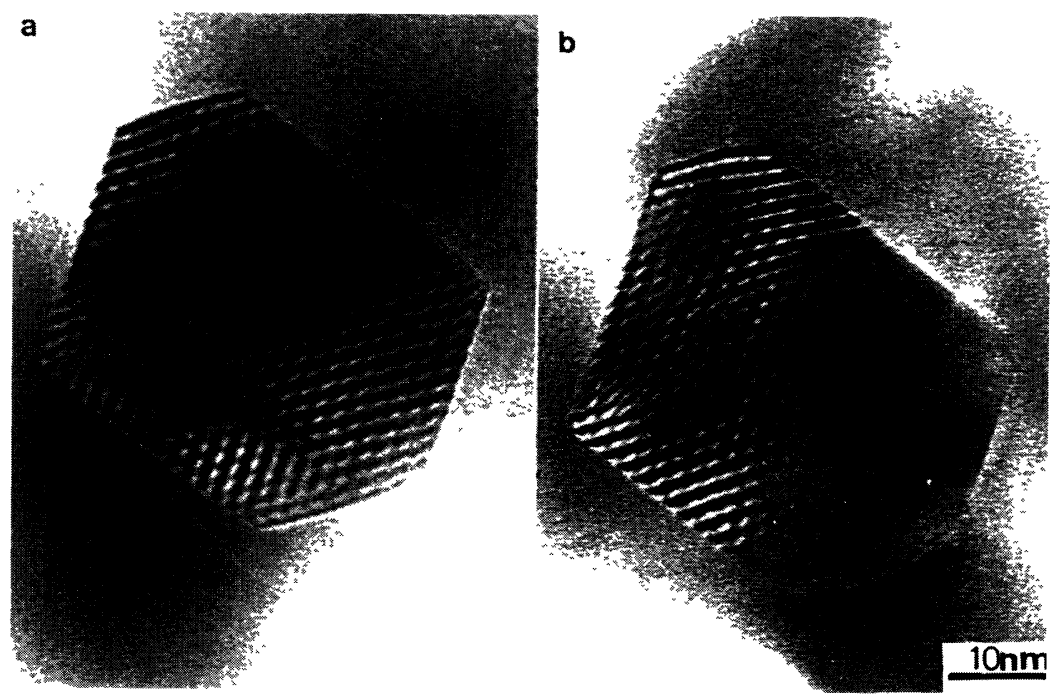

Fig. 1. - High resolution images of the melting process of an In particle viewed along the $\langle 110\rangle$ direction. Moire' fringes which are observed in the whole area (a) and in the left hand side of the In particle (b) indicate the area of solid In.

\section{Results.}

The In particles were cuboctahedral in shape bounded by eight $\{111\}_{\mathrm{Al} \text {, In }}$ and six $\{100\}_{\mathrm{Al} \text {, In }}$ facets as shown schematically in figure 2 . In many cases, one void was observed on one of the $\{100\}_{\mathrm{Al}}$, In facets of each particle. During the heating experiment, the sample was heated and cooled at a heating and cooling rate in the range $1 \sim 2 \mathrm{~K} / \mathrm{hr}$ around the melting temperature of In particles. After the heating experiment for about $10^{5}$ seconds, there was no change in either the In particle shape or the orientation relationship between the In particles and the Al matrix [6,9]. The melting process of In particles proceeds in six stages, at each of which one of the $\{100\}$ facets becomes covered successively with the liquid phase as illustrated in figure 2 . Initially, the liquid droplet is nucleated at a $\{100\}$ facet or at the cavity which is in contact with one of the $\{100\}$ facets (Fig. 2a), and repeatedly expands and shrinks as shown in figure 3 (Stage 1). The liquid droplet shows the most shrinkage in the shape viewed along a $<110>$ and $<100>$ direction in figures $3 a$ and $3 c$, respectively. The most expansion of the liquid droplet viewed along the $\langle 110>$ direction is shown in figure $3 b$. The interface between solid and liquid $\operatorname{In}\left(\operatorname{In}_{\mathrm{s}} / \operatorname{In}_{1}\right)$ showed a flip-flop like motion between two shapes. The time spent in this stage was longest in the whole process of melting. Stage 2 (Fig. 2b): the hemispherical $\mathrm{In}_{\mathrm{s}} / \mathrm{In}_{1}$ interface started to propagate into the interior of the In particle, and reached one of the four nearest $\{100\}$ facets as shown in figure 4a. Stage 3 (Fig. 2c): the liquid phase expanded further and covered the other of the nearest $\{100\}$ facets. Stage 4 (Fig. 2d): the $\mathrm{In}_{\mathrm{s}} / \mathrm{In}_{1}$ interface was anchored at the two vertices at each of which two $\{111\}$ facets and a $\{100\}$ facet come together and bowed out (Fig. 4b). Stage 5 (Fig. 2e): one of the last two $\{100\}$ facets which had not been covered with the liquid phase now became covered with the liquid phase, and the $\operatorname{In}_{s} / \mathrm{In}_{1}$ interface became parallel to the $\{100\}$ facet (Fig. $4 \mathrm{c}$ ). Stage 6 (Fig. 2f): the $\mathrm{In}_{\mathrm{s}} / \mathrm{In}_{1}$ interface propagated, while keeping the interface parallel to the $\{100\}$ plane, until the whole volume of the In particle melted. These melting processes were observed in the particles whose $\{100\}$ facet separations are larger than $100 \AA$. These intermediate stages of melt- 
ing in the particle smaller than $100 \AA$ have not been observed. The difference of the melting process between particles larger than $100 \AA$ and those smaller than $100 \AA$ is also suggested by the relation between superheating temperature $(\Delta T)$ and reciprocal length of the $\{100\}$ facet separation $(1 / L)[8]$ as shown in figure 5. $\Delta T$ s of the particles smaller than $100 \AA$ are on the straight line through the original point with a slop $\Delta T L=2000 \AA \mathrm{K}$. The straight line, however, could not be drawn for the $\Delta T$ s of the particles larger then $100 \AA$.
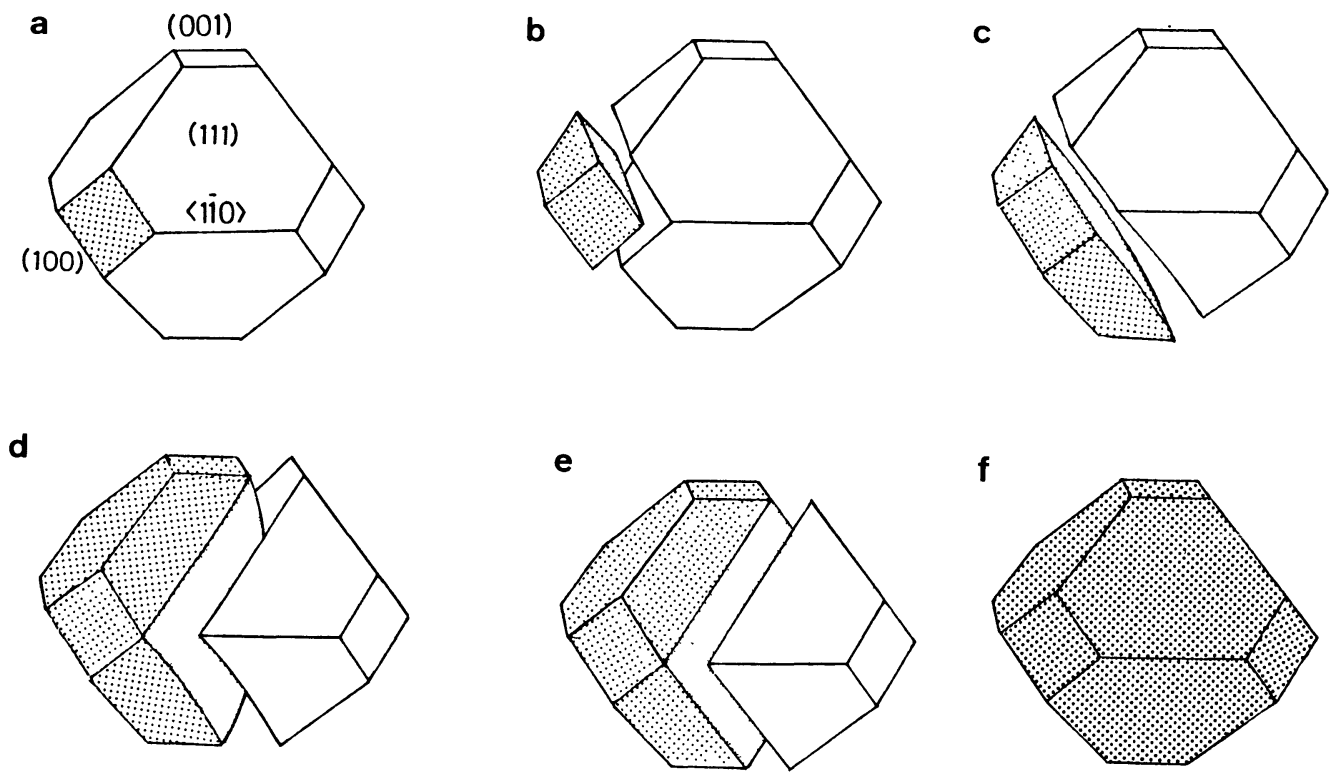

Fig. 2. - Schematic diagrams of the melting process of the In particle embedded in an $\mathrm{Al}$ matrix. Hatching indicates the liquid of In.

\section{Discussion.}

The relation among the interfacial energy per unit area of the interfaces concerning the melting process could be determined as the result of the detailed observation of the process. The relationship between the interfacial energy per unit area of the interface between soldi $\mathrm{Al}$ an In on the $\{100\}\left(\sigma_{1}\right)$ and the $\{111\}\left(\sigma_{2}\right)$ facet can be written as $h_{100} / \sigma_{1}=h_{111} / \sigma_{2}$ using by Curie-Wulff theorem. Where $h_{100}$ and $h_{111}$ are the one half of the $\{100\}$ and $\{111\}$ facet separation, respectively. Substituting the value of $k \equiv h_{100} / h_{111}$ measured from figure $1 \mathrm{a}$, we obtain $\sigma_{1}=1.20$ $( \pm 0.06) \sigma_{2}$. The interface between solid $\mathrm{Al}$ and liquid In parallel to $\{111\}$ persists after the In particle becomes molten completely. That means that the interfacial energy per unit area on this plane $\left(\sigma_{3}\right)$ is much lower than that on the plane with other orientations $\left(\sigma_{4}\right)$. The relative values of the interfacial energies per unit area $\sigma_{1}, \sigma_{2}, \sigma_{3}, \sigma_{4}$ and those of the $\operatorname{In}_{\mathrm{s}} / \operatorname{In}_{1}$ interface $\left(\sigma_{5}\right)$ were determined from the contact angles between interfaces during the melting process as follows.

In stage 1 , a droplet of liquid $\mathrm{In}$ is nucleated on a $\{100\}$ solid $\mathrm{Al} / \mathrm{In}$ interface. As illustrated in 

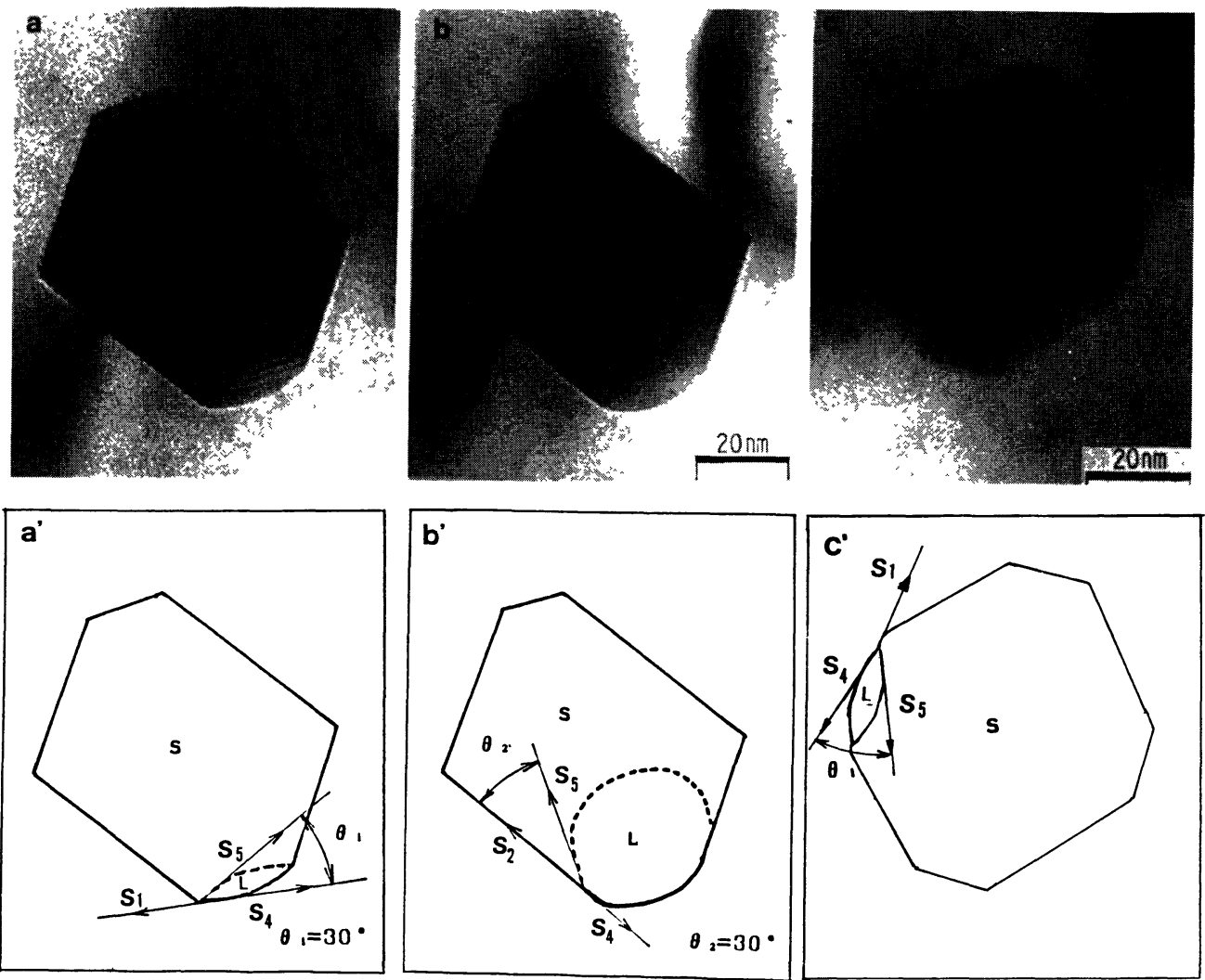

Fig. 3. - TEM micrographs of the melting process of an In particle viewed along [011] (a,b) and [001] (c) and schematic diagrams (a', b') and (c') corresponding to $(a, b)$ and (c), respectively. The areas indicated by $S$ and $L$ correspond to solid and liquid In, respectively.

figure 3a', the relation among $\sigma_{1}, \sigma_{4}$ and $\sigma_{5}$ can be written by

$$
\sigma_{4}+\sigma_{5} \cos \theta_{1}=\sigma_{1}
$$

where $\theta_{1}$ is the contact angle between the $\mathrm{In}_{\mathrm{s}} / \mathrm{In}_{1}$ interface and the interface between solid $\mathrm{Al}$ and liquid In, which is measured from figure $3 \mathrm{a}$ to be $30^{\circ} \pm 10^{\circ}$. This relation is more apparent in figure 3c. The droplet is not expanding fully on the $\{100\}$ facet, so that we can observe that the interface between solid $\mathrm{Al}$ and $\mathrm{In}$ on the $\{100\}$ plane is in a balance against the $\mathrm{In}_{\mathrm{s}} / \mathrm{In}_{1}$ interface and the interface between solid $\mathrm{Al}$ and liquid $\mathrm{In}$ as illustrated in figure $3 \mathrm{c}$. The geometrical relation between figures $3 \mathrm{a}$ and $3 \mathrm{c}$ is illustrated in figure 6 schematically. At the largest bowing out of the In droplet in stage 1 , as illustrated in figure $3 \mathrm{~b}$, the relation among $\sigma_{2}, \sigma_{4}$ and $\sigma_{5}$ can be written by

$$
\sigma_{4}=\sigma_{2}+\sigma_{5} \cos \theta_{2}
$$

where $\theta_{2}$ is the contact angle between the $\mathrm{In}_{\mathrm{s}} / \mathrm{In}_{1}$ interface and the interface between solid $\mathrm{Al}$ and In on the $\{111\}$ plane, which is measured from figure $3 \mathrm{~b}$ to be $30^{\circ} \pm 10^{\circ}$. In stage 4 , as illustrated in figure $4 \mathrm{c}^{\prime}$, the relation among $\sigma_{3}, \sigma_{2}$ and $\sigma_{5}$ can be written by

$$
\sigma_{3}=\sigma_{2}+\sigma_{5} \cos \theta_{3}
$$



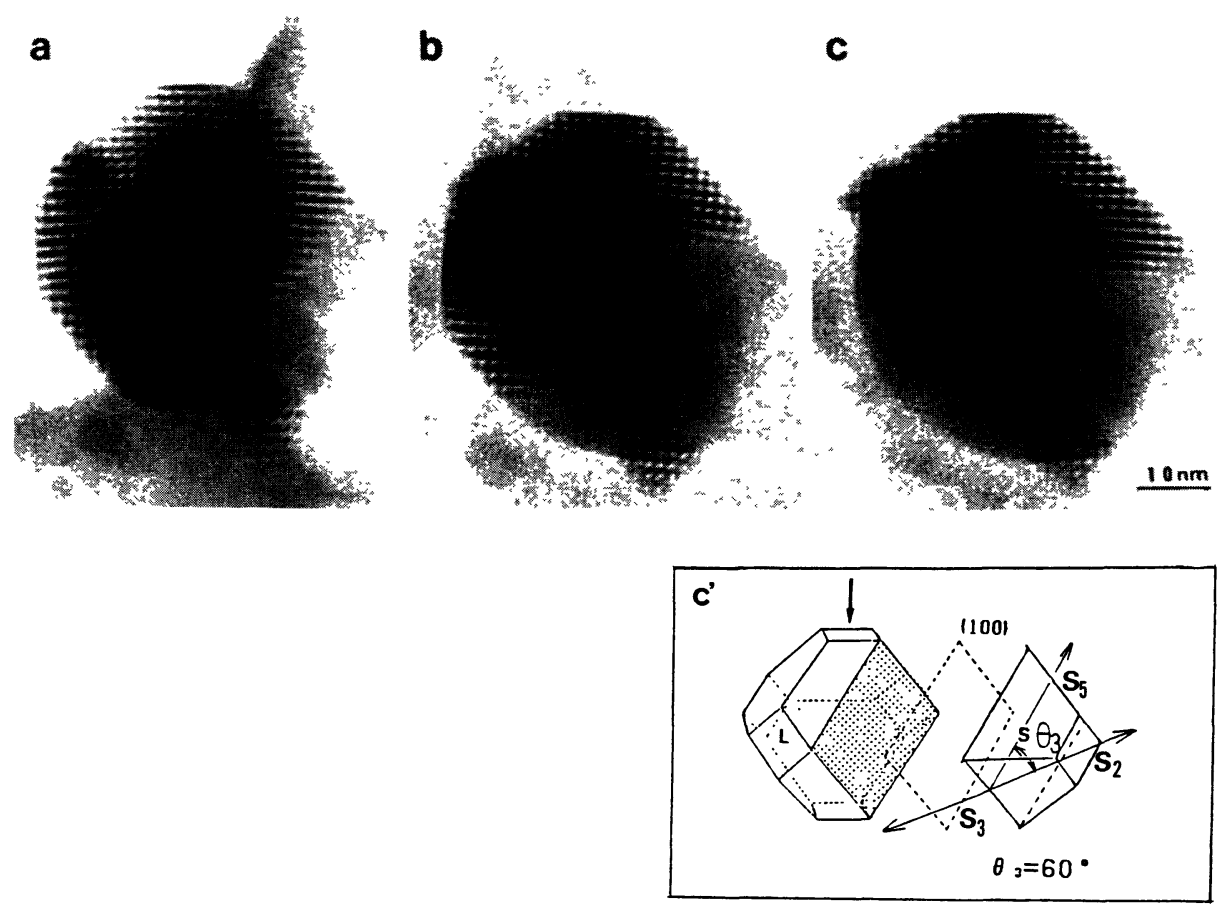

Fig. 4. - TEM micrographs of the melting process of an In particle viewed along [001] $(a, b, c)$ and a schematic diagram (c') corresponding to (c). The areas indicated by $S$ and $L$ correspond to solid and liquid In, respectively.

where $\theta_{3}$ is the contact angle between the $\operatorname{In}_{\mathrm{s}} / \operatorname{In}_{1}$ interface and the interface between solid $\mathrm{Al}$ and liquid In on $\{111\}$, which is measured from figure $4 \mathrm{c}$ to be $60^{\circ} \pm 10^{\circ}$. Equations $(1,2)$ and (3) give the following relation among $\sigma_{1}, \sigma_{2}, \sigma_{3}, \sigma_{4}$ and $\sigma_{5}$.

$$
\begin{aligned}
\sigma_{1}=1.20( \pm 0.06) \sigma_{2}>\sigma_{4} & =1.105( \pm 0.038) \sigma_{2}>\sigma_{3}=1.067( \pm 0.042) \sigma_{2}>\sigma_{2} \\
& >\sigma_{5}=0.122( \pm 0.048) \sigma_{2}
\end{aligned}
$$

Allen, Gile and Jesser [10] analyzed the superheating temperature $\Delta T$ of particles embedded in a matrix by thermodynamic theory [4]. They describe the relation between the $\Delta T$ and the radius of the particle $(r)$ using the model of spherical shape particles as follows

$$
\Delta T=\left(T_{m} / \Delta H\right)\left\{3\left(\sigma_{\mathrm{s}}-\sigma_{\mathrm{L}}\right) / r-\Delta E\right\}
$$

where $T_{m}$ is the melting temperature of bulk In, $\Delta H$ the latent heat, $\Delta E$ the change in strain energy density on melting, $\sigma_{\mathrm{s}}$ and $\sigma_{\mathrm{L}}$ the interfacial energy per unit area between the particle and matrix before and after melting, respectively. According to equation (5), the relation between $\Delta T$ and $1 / r$ will draw a straight line. That is corresponding to the region of the particles smaller than $100 \AA$ as shown in figure 5. Now we know not only the detailed shape on the In particle but also the relation among the interfaces concerning the melting process. This means that the assumption of the spherical shape of the particle is not required and the detail of $\sigma_{\mathrm{s}}, \sigma_{\mathrm{L}}$ can be written. The interfacial energy between solid $\mathrm{Al}$ and In $\left(4 \pi r^{2} \sigma_{\mathrm{s}}\right)$ can be written by

$$
4 \pi r^{2} \sigma_{\mathrm{s}}=A_{1} \sigma_{1}+A_{2} \sigma_{2}
$$




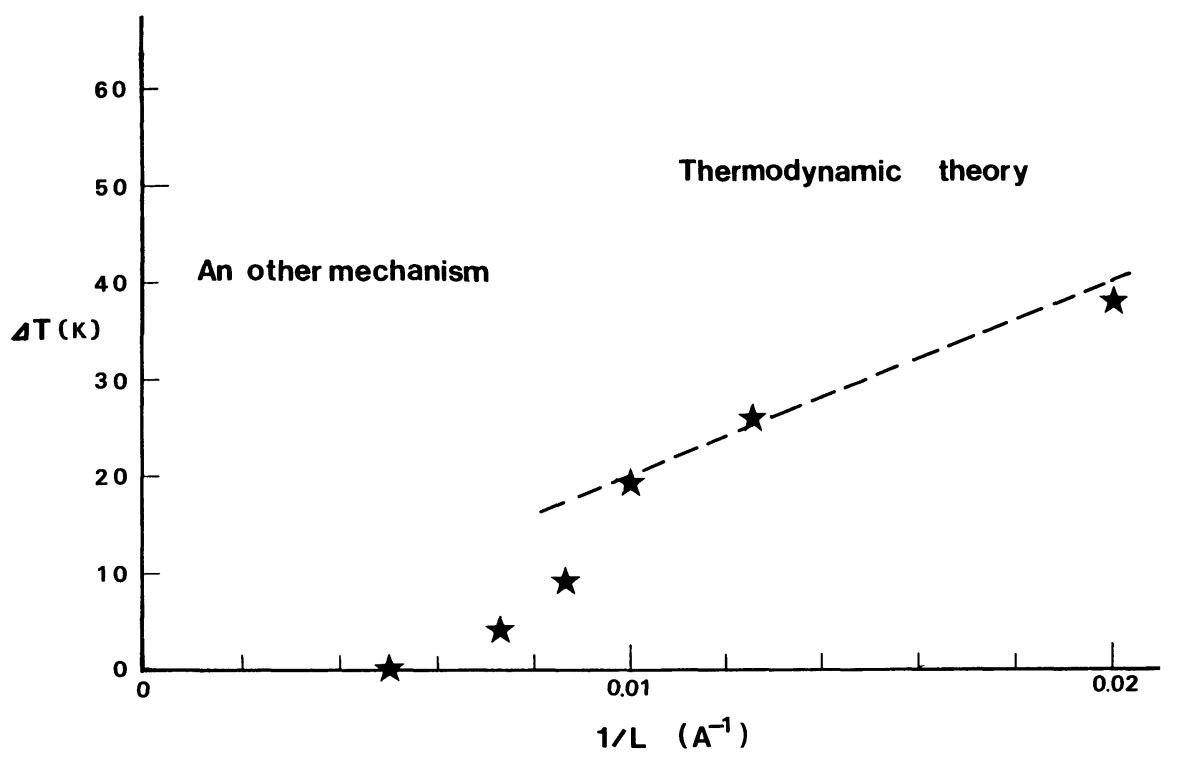

Fig. 5. - The relation between superheating temperature $(\Delta T)$ and reciprocal length of the $\{100\}$ facet separation $(1 / L)[8]$. The $\Delta T s$ of the particle smaller than $100 \AA$ are on the straight line through the original point with a slop $\Delta T L=2000 \AA \mathrm{K}$.

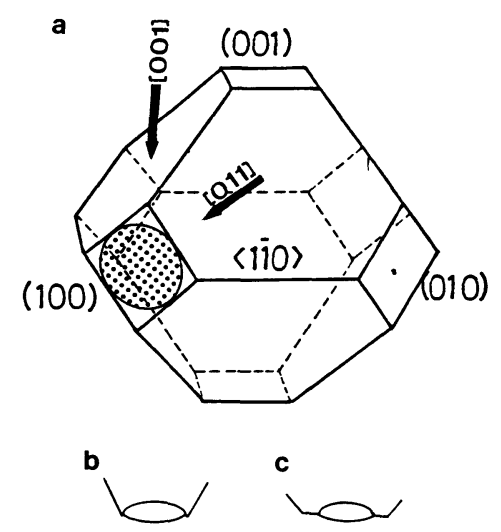

Fig. 6. - Schematic illustration of the relation between the shape of the liquid droplet (hatching in (a)) of In on the (100) facet viewed along [011] (Fig. 3a) and [001] (Fig. 3c). Although the droplet does not expand full of the (100) facet (a), the [011] projection (b) shows that the (100) facet is covered by liquid In, completely. The solid Al and In interface which surrounds the liquid droplet on the (100) facet can be observed in the [001] projection (c).

where $A_{1}$ and $A_{2}$ are the area of the interface between solid $\mathrm{Al}$ and In on $\{100\}$ and $\{111\}$, respectively. These two areas $A_{1}$ and $A_{2}$ can be calculated as a simple geometrical problem of cuboctahedron. The specific lengths from $l_{0}$ to $l_{6}$ of cuboctahedral In particle, which will help the calculation of $A_{1}$ and $A_{2}$, are shown in figure 7a. All these lengths can be described using by the $\{100\}$ facet separation $L\left(=2 h_{100}\right)$ and the ratio between the $\{100\}$ and $\{100\}$ facet separation 
$k\left(=h_{100} / h_{111}\right)$. The geometrical representation of the calculation of $A_{1}$ and $A_{2}$ are illustrated in figures $7 \mathrm{~b}$ and $7 \mathrm{c}$, respectively. $A_{1}$ and $A_{2}$ can be written by
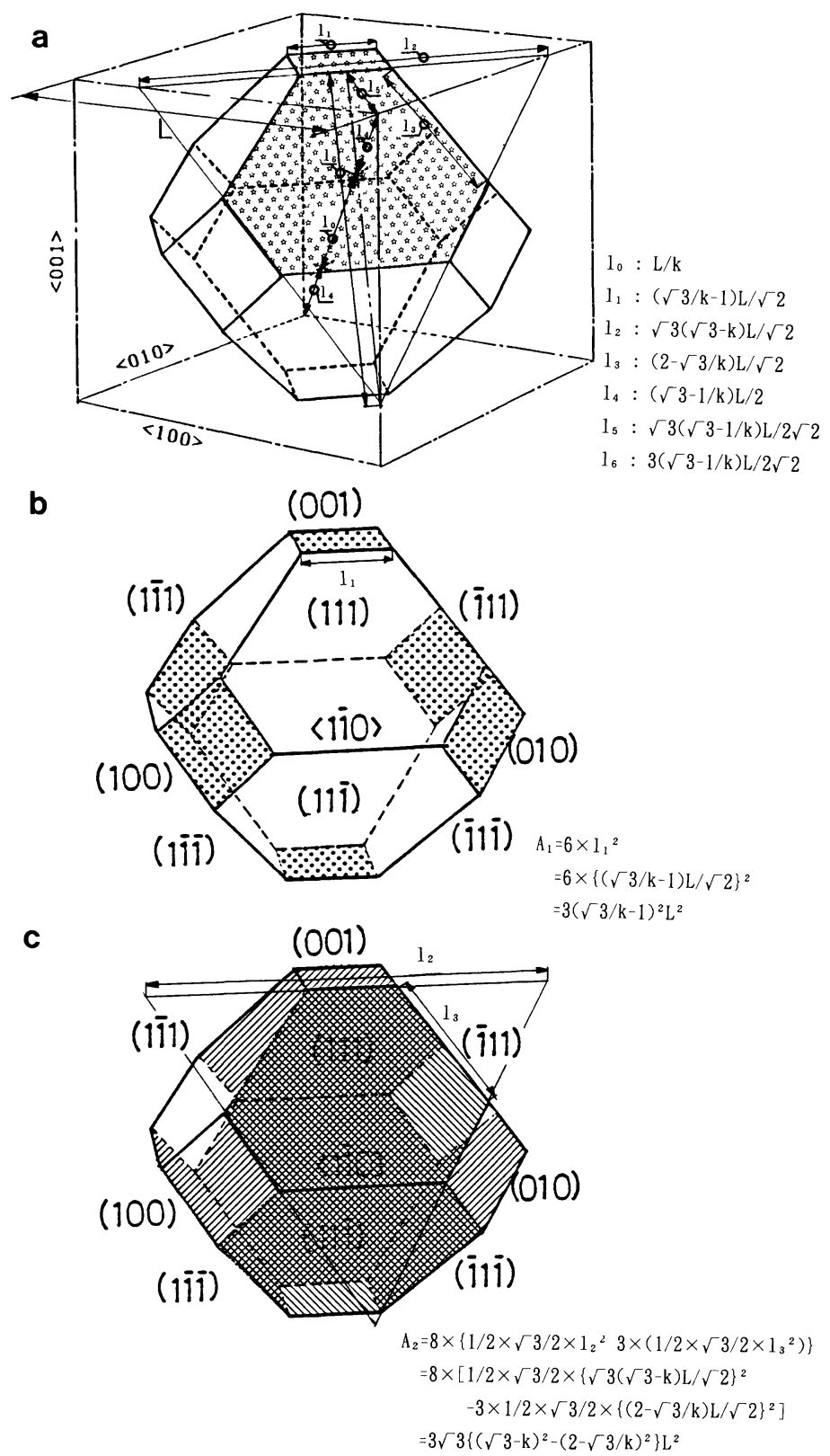

Fig. 7. - Schematic representation of the geometrical property of cuboctahedron. The lengths from $l_{0}$ to $l_{6}$ can be described by $L$ and $k(\mathrm{a})$. The area of $A_{1}$ is six times of the area of a $\{100\}$ facet, which can be written by $l_{1}^{2}$ (b). The area of $A_{2}$ is eight times of the area of a $\{111\}$ facet, which can be written by $1 / 2 \times \sqrt{ } 3 / 2 \times l_{2}^{2}-3 \times\left(1 / 2 \times \sqrt{ } 3 / 2 \times l_{3}^{2}\right)(c)$. 


$$
\begin{aligned}
A_{1} & =6 l_{1}^{2}=3(\sqrt{ } 3 / k-1)^{2} L^{2} \\
A_{2} & =8 \times\left\{1 / 2 \times \sqrt{ } 3 / 2 \times l_{2}^{2}-3 \times\left(1 / 2 \times \sqrt{ } 3 / 2 \times l_{3}^{2}\right)\right\} \\
& =3 \sqrt{ } 3\left\{(\sqrt{ } 3-k)^{2}-(2-\sqrt{ } 3 / k)^{2}\right\} L^{2}
\end{aligned}
$$

The interfacial energy between solid $\mathrm{Al}$ and liquid $\mathrm{In}\left(4 \pi r^{2} \sigma_{\mathrm{L}}\right)$ can be written by

$$
4 \pi r^{2} \sigma_{\mathrm{L}}=A_{3} \sigma_{3}+A_{4} \sigma_{4}
$$

where $A_{3}$ and $A_{4}$ are the area of the interface between solid $\mathrm{Al}$ and liquid In on $\{111\}$ and other plane, respectively. The area of $A_{3}$ and $A_{4}$ are approximately equal to $A_{2}$ and $A_{1}$, respectively. The volume of a particle $V_{0}$ can be written by

$$
V_{0}=\left\{1-\sqrt{ } 3 / 2(\sqrt{ } 3-k)^{3}+(2-\sqrt{ } 3 / k)^{3} / 2\right\} L^{3}
$$

If we put

$$
\begin{aligned}
& a_{1} \equiv 3(\sqrt{ } 3 / k-1)^{2}, a_{2} \equiv 3 \sqrt{ } 3\left\{(\sqrt{ } 3-k)^{2}-(2-\sqrt{ } 3 / k)^{2}\right\} \\
& v_{0} \equiv\left\{1-\sqrt{ } 3 / 2(\sqrt{ } 3-k)^{3}+(2-\sqrt{ } 3 / k)^{3} / 2\right\}
\end{aligned}
$$

Equation (5) can be rewritten using by equations $(6,7,8,9)$ and $(10)$ as follow

$$
\Delta T=-\left(T_{m} / \Delta H\right)\left\{\left(\sigma_{1} a_{1}+\sigma_{2} a_{2}\right)-\left(\sigma_{3} a_{2}+\sigma_{4} a_{1}\right)\right\} / v_{0} L-\Delta E
$$

The corresponding value of $L$ in spherical model is the diameter $2 r(=0.91 L)$ of the particle. In the region of the $L$ is less than $100 \AA$ where the thermodynamic theory [4] is controlling the melting of the particle, we can put the value of $\Delta T L=2000 \AA \mathrm{K}$ into equation (11). The value of the bulk In of $\Delta H$ was used for the calculation. As the result of the substitution of equation (4) into equation (11), the absolute values of the interfacial energy per unit area $\sigma_{1}, \sigma_{2}, \sigma_{3}, \sigma_{4}$ and $\sigma_{5}$ were determined as follows

$$
\begin{aligned}
& \sigma_{1}=257 \pm 115\left(\mathrm{erg} / \mathrm{cm}^{2}\right) \\
& \sigma_{2}=242 \pm 121\left(\mathrm{erg} / \mathrm{cm}^{2}\right) \\
& \sigma_{3}=253 \pm 124\left(\mathrm{erg} / \mathrm{cm}^{2}\right) \\
& \sigma_{4}=231 \pm 99\left(\mathrm{erg} / \mathrm{cm}^{2}\right) \\
& \sigma_{5}=31 \pm 20\left(\mathrm{erg} / \mathrm{cm}^{2}\right)
\end{aligned}
$$

The interfacial energy per unit area of the interface between solid $\mathrm{Al}$ and liquid $\mathrm{In}$ determined by dihedral angle method [11] to be $322 \pm 24 \mathrm{erg} / \mathrm{cm}^{2}$, which can be calculated by equation (14) in reference is in good agreement with the value of $\sigma_{3}$ and $\sigma_{4}$. The interfacial energy per unit area on $\mathrm{In}_{\mathrm{s}} / \mathrm{In}_{1}$ interface $\sigma_{5}$ also shows good agreement with the value determined by Allen et al. [12].

These agreements mean the following results.

1) That is a proof of that the thermodynamic theory [4] is controlling the melting process of the particle smaller than $100 \AA$. 
2) The value of bulk material of $\Delta H$ can be applied to the calculation means that the difference of the pressure due to the volume change on melting which will cause the change of latent heat $\Delta H$ and also strain energy density $\Delta E$ on melting is negligible. That is presumably due to the presence of the void contacting the particle. The formation of the void can be understood when we take into account the process of solidification of this material. In the $\mathrm{Al}$-In phase diagram [7], $\mathrm{Al}$ solidifies at $912 \mathrm{~K}$ and $\mathrm{In}$ rich liquid is left in the $\mathrm{Al}$ matrix at the composition of our sample. The In rich liquid will deposit $\mathrm{Al}$ onto the interface between solid $\mathrm{Al}$ and the liquid between $912 \mathrm{~K}$ and $450 \mathrm{~K}$. At the melting point of In, pure In liquid will be left in the Al matrix. The volume change $\left(V_{\mathrm{s}}-V_{1}\right) / V_{1}=-2.6 \%$ on solidification of In causes the void which is in contact with the In particle. That void would have enough volume to cancel the volume change on melting of the In particle.

3) The step by step propagation of the melting of In into the particle (six stages of melting process) lowers the superheating temperature of the particle larger than $100 \AA$.

\section{Conclusions.}

The melting process of In particles embedded in an $\mathrm{Al}$ matrix, which was prepared by splat quenching, has been observed, continuously. The interfacial energies of solid-liquid interface of In, the interfaces between solid $\mathrm{Al}$ and $\mathrm{In}$, and solid $\mathrm{Al}$ and liquid In were thermodynamically analyzed, and the following results have been obtained.

1) In the region of the particle larger than $100 \AA$, melting started either at a cavity or at one of the $\{100\}$ facets and proceeded into the interior of the In particle in six stages in such a way that, at each of the stages, one of the $\{100\}$ facets became covered with the liquid phase. These processes lower the superheating temperature of the particles compared to the particles smaller than $100 \AA$.

2) In the region of the particle smaller than $100 \AA$, these intermediate stages of the melting have not been observed and the relation between superheating temperature and the particle diameter obeys the thermodynamic theory [4].

3) The absolute values of interfacial energy per unit area for all interfaces concerning the melting process were determined.

4) The effect of volume change on melting process is negligible in the case of the In particles embedded in an $\mathrm{Al}$ matrix.

\section{Acknowledgements.}

This work was supported by the Grant-in-Aid for Encouragement of Young Scientists No.04750604 from Ministry of Education on Science and Culture and the Kazato Research Foundation.

\section{References}

[1] Moore K., Chattopadhyay K. and Cantor B., Proc. R. Soc. Lond. A414 (1987) p. 499.

(2) TAKAGi M., J. Phys. Soc. Jpn. 9 (1954) p. 359.

[3] Berman R. and Curzon A., Canadian J. Phys. 52 (1974) p. 923.

[4] COUCHMAN P. and JESSER, Nature 269 (1977) p. 481.

[5] SKripoV V., KoverdA V. and SKoKoV V., Phys. Stat. Sol. A66 (1981) p. 109.

[6] SASAKI K. and SaKa H., Phil. Mag. A63 (1991) p. 1207. 
[7] "Binary Alloy Phase Diagrams”, Eds. T. Massalski, J. Murray, L. Bennett and H. Baker, Am. Soc. Metals (Metals Park, 1986).

[8] SaKa H., NishiKaWA Y. and ImURA T., Phil. Mag. A57 (1988) p. 895.

[9] Zhang D. and CANTON B., Phil. Mag. A62 (1990) p. 557.

[10] Allen G., GiLE W. and JeSSER W., Acta Metall. 28 (1980) p. 1695.

[11] CAMEl D., Eustathopoulos N.E. and DeSRe P., Acta Metall. 28 (1980) p. 239.

[12] Allen G., BAyles R., Gile W. and JesSER W., Thin Solid Films 144 (1986) p. 297. 